\title{
Salud Infantil en Chile y el Rol de la Colaboración Internacional
}

\author{
Child Health in Chile and the role of the international collaboration
}

\begin{abstract}
Important progress has been made in child health in Chile in recent years. This is shown in the reduced rates of infant and early childhood mortality which in 1981 were $27.2 \%$ and $1.6 \%$ respectively.

This change is due to the country's global development as well as to the successful child and family health programs characterized by their broad coverage. The main features of these achievements are discussed.

A résumé is made of the technical assistance provided through international cooperation with special emphasis on that given by the World Health Organization, the Pan American Health Organization and UNJCEF. This assistance has contributed in a great measure to the support and improvement of the national health activities.
\end{abstract}

Los problemas propios de la salud infantil afectan a grupos etarios definidos, los que pueden en ciertos medios sufrir el impacto del subdesarro. llo económico-social y cultural, el incremento demográfico acelerado y la dificultad consiguiente de poder disponer de servicios sociales adecuados, incluidos los de índole sanitaria.

Dicha situación determina riesgos que influyen en los procesos de crecimiento y desarrollo $y$ eventualmente en las condiciones de salud y bienestar ulteriores. (1 a 6 ).

Los niños y los jóvenes necesitan protección especial, tanto en los aspectos ligados a su adaptación armónica al medio ambiente, como en tomo a su preparación para una vida que les permita participar con eficiencia en el desarrollo nacional (3).

En la actualidad está encarnado en la conciencia colectiva de los países, el imperativo de dar cum. plimiento a las resoluciones establecidas tanto en la Declaración Universal de los Derechos del Hombre, que deben traducirse fundamentalmente en la obtención de un adecuado nivel de vida, salud $y$ bienestar $y$ que incluye el cuidado $y$ asistencia especiales durante la infancia(7), como en las que se explicitan en la Declaración de

* Ex-Asesor Regional en Salud Maternoinfantil en la Organización Panamericana de la Salud (OPS/OMS) y en el Instituto Interamericano del Niño (OEA).
Naciones Unidas sobre Derechos de los Niños (8), y que con la pertinente adaptación, se pasan a reseñar.

- Derechos aplicables a todos los Niños

- Derecho al Nombre y a la Nacionalidad

- Derecho a la Seguridad Social y a la Salud

- Derecho al Desarrollo Integral Normal

- Derecho al Afecto Familiar

- Derecho a la Educación y Recreación

- Derecho a la Readaptación del Nif́o Especial

- Derecho preferente de Socorro

- Derecho de Protección Laboral

- Derecho a la Convivencia Social

La responsabilidad, tanto en la implementación de los derechos mencionados, como en la satisfacción de las necesidades de los niños, compete a la familia, a la comunidad, al Estado, y en singular medida a la colaboración internacional sistematizada (3).

Por ser los requerimientos pertinentes planteados por el niño y el joven de indole polifacética -entre los que cabe subrayar los relacionados con la salud, la alimentación, la educación y el bienestar social, incluidas las oportunidades recreacionales y laborales - el cumplimiento de los postulados a que se ha hecho referencia, exige una decidida voluntad política que debe traducirse en la promoción de una coordinada ejecución de programas intersectoriales a cargo de los organismos gubernamentales y privados correspondientes, contando con una permanente y activa participación de las 
familias y de las comunidades a beneficiar (3).

Precisamente y con motivo del Año Internacional del Niño (9), importante evento patrocinado por las Naciones Unidas y llevado a cabo en 1979 , se ha efectuado a nivel mundial y en los distintos países un exhaustivo analisis de la problemática que enfrenta la salud y el bienestar del niño y su familia, pudiéndose por estas circunstancias contribuir en forma más adecuada a la formulación de planes y de programas y a la realización de actividades específicas en el contexto de las resoluciones pertinentes de la Organización Mundial de la Salud (10) y de Naciones Unidas (11) destinadas a lograr la obtención hacia el año 2000, de Salud para todos los ciudadanos del mundo, para que puedan llevar una vida social y económicamente productiva. Ello lleva implicado el imperativo de asignar una prioridad destacada a todo lo referente a la salud infantil y familiar (5).

En la Región de las Américas, son a dicho respecto de un inapreciable valor, los estudios realizados por UNICEF (12) y la Organización Panamericana de la Salud (13).

A la luz de las premisas expuestas, cabe destacar que en Chile, guardando algún paralelismo con lo ocurrido en distintos países, en particular de Latinoamérica y el Caribe, se ha estado registrando en las décadas más recientes, una tendencia prácticamente continua hacia la obtención progresiva de condiciones más adecuadas de salud y bienestar en sectores cada vez más amplíos de la comunidad.

Aún cuando los recursos y el quehacer nacional han sido decisivos en la obtención de los resultados mencionados, puede estimarse con fundamento que ha ejercido en dicho fenómeno una influencia catalítica de singular provecho, la cooperación técnica que en áreas especificas, han brindado los Organismos de Colaboración Internacional, sean éstos de tipo bi o multilateral.

Precisamente, el análisis y la ponderación de los hechos reseñados y acaecidos en Chile, apunta a la finalidad del presente estudio que pasa a exponerse.

\section{SITUACION DE LA SALUD INFANTO-JUVENIL EN CHILE}

La situación de la salud del niño y del joven en Chile, como en otros medios, es el resultado de las condiciones sanitarias generales de la población, las que a su vez se influyen por diversos factores de índole demográfico, económico-social y cultural, que se han estado modificando, en un variable contexto político en el último tiempo. Estimamos a dicho respecto que es procedente proporcionar una reseña general de algunos de los parámetros mencionados de acuerdo a información recopilada mayormente por el Banco Interamericano de Desarrollo (14).

En fecha reciente la población del pais ascendía a 11.114 .000 habitantes, de los cuales el $32.6 \%$ era menor de 15 años de edad, alcanzando a $61.9 \%$ y $5.5 \%$ los grupos etarios entre 15 y 65 años y mayores de esa edad respectivamente (15).

La distribución poblacional acusa un notorio predominio urbano $(81.5 \%)$, el que se ha determinado por los significativos procesos de migración del campo a las ciudades ocurridos en los últiunos años. Por otra parte, desde mediados de la década del 60 , se ha registrado una disminución del crecimiento vegetativo, el que descendió desde $2.47 \%$ en 1960 , hasta $1.70 \%$ en 1981 , como resultado fundamentalmente del descenso de las tasas de natalidad y de fecundidad, que son en la actualidad del orden de $23.2 \%$ (16) y $2.9 \% 0$ respectivamente.

Finalmente y en el ámbito de la dinámica demografica, cabe destacar que la expectativa de vida alcanza a 65.7 af́os en el quinquenio más reciente, como resulțado básico del descenso de las tasas de mortalidad general desde $9.2 \% 0(1972)$ a $6.2 \% 0$ (1981).

El desarrollo económico-social del país ha sido -salvo situaciones coyunturales transitorias- progresivo, pudiéndose traducir, utilizando parámetros convencionales internacionales, aunque no plenamente rigurosos, en un promedio por habitante, en 1980 de \$E.U.A. 1.590 (14). Sin embargo, su distorsionada distribución, determina la existencia de estratos económicosociales muy diferentes, debiendo a este respecto, señalarse que hasta un $6 \%$ y un $17 \%$ de los hogares se encuentran en situaciones de indigencia y de pobreza crítica respectivamente (17).

Por otra parte, cabe mencionar que la tasa de alfabetización ha aumentado notoriamente, siendo de $94 \%$ en 1980 y alcanzando la tasa de escolarización, entre niffos de 6 a 11 af́os, a $93 \%$ (18).

Asimismo debe destacarse que otros importantes indicadores de desarrollo social, estrechamente ligados al bienestar, tales como la alimentación y el saneamiento ambiental, revelan perfiles susceptibles de superar. A dicho respecto cabe señalar que la disponibilidad alimentaria en fechas recientes, ha sido del orden de 2540 calorías y 61.3 y 27.2 gramos de proteina total $y$ de ongen animal respectivamente (13). En lo referente a sanea. miento del medio, aún cuando se han registrado últimamente progresos, éstos han sido restrictos, en especial en áreas rurales. La situación pertinente revela que en la actualidad, el $72 \%$ y el $14 \%$ de las poblaciones urbana y rural cuentan con disponibilidad de agua potable, siendo la problemática más precaria en relación a la eliminación de excretas, 
ya que sólo el $56 \%$ dispone de alcantarillado en el medio urbano, en tanto que a nivel rural, únicamente el $12 \%$ cuenta con letrinas sanitarias (19).

Todo parece indicar que es necesario poseer indicadores globales que razonablemente $y$ en forma sintética, expresen los niveles de desarrollo nacional y/o regional que tan ligados se encuentran a la fisonomia sanitaria. Esta ha sido una preocupación primordial, tanto de los países, como de las organizaciones correspondientes de colaboración internacional. A este respecto cabe mencionar las investigaciones del Consejo de Desarrollo de Ultramar de Estados Unidos de América (20), acogidas con gran interés por UNICEF (21) y puestas en práctica en el continente, con el estímulo y apoyo de la Oficina Regional para las Américas del Fondo de Naciones Unidas para la Infancia (18). Los nuevos indicadores que los estudios en referencia proponen, permiten la expresión de programas sociales amplios y reflejan aspiraciones universales al través de "indices que se identifican con la calidad física de la vida" (ICFV) y que ponderan tanto la esperanza de vida, como las tasas de mortalidad infantil y de alfabetismo (20, 21), estableciéndose rangos que van de 0 a 100 , equivalente esta última cifra a 77 años, $7 \% 0 \mathrm{y}$ $100 \%$ respectivamente. En este mismo orden de ideas, debe referirse a la cuantificación porcentual de la modificación en el tiempo, de los parámetros reseñados, utilizando las "tasas de reducción de la disparidad (TRD), con el propósito de ponderar la mejoría que se haya podido obtener en la calidad vital, cuyas metas en el contexto de la "Declaración de Amsterdam" (21), postulan valores del orden de un $3.5 \%$ anual, hasta el año 2000 y las que podrían ser consideradas con provecho en la evaluación de los Planes de Acción pertinentes de la Organización Mundial (22) y Panamericana de la Salud (23).

En la tabla 1, se expone la situación pertinente de Chile, en lo que se refiere a la determinación de los indicadores antes mencionados.

Tabla 1

Indices de Calidad Física de la Vida (ICFV) y Tasas de Reducción de Disparidad(TRD) Anual Chile. 1956-1979*

\begin{tabular}{|c|c|c|c|c|c|c|c|c|}
\hline \multirow[t]{2}{*}{ Años } & \multirow{2}{*}{\multicolumn{2}{|c|}{ ICTV TRD }} & \multicolumn{2}{|c|}{ Expectativa Vital } & \multicolumn{2}{|c|}{ Mortalidad Infantil } & \multicolumn{2}{|c|}{ Alfabetismo } \\
\hline & & & Аกิด & TRD\% & $\%$ & $\operatorname{TRD} \%$ & $\%$ & TRD $\%$ \\
\hline $1950 / 59$ & 61.9 & $\therefore-$ & 58.7 &.-- & 112.4 &.-- & 80.2 &.-- \\
\hline $1970 / 79$ & 77.1 & 2.8 & 67.5 & 3.0 & 78.8 & 2.2 & 88.1 & 2,8 \\
\hline
\end{tabular}

*Fuente: Grant, \$.P. (Ref, 20).

La situación ecológica anteriormente expuesta, influye notoriamente en las condiciones de salud en general $y$ en especial en la de los niños. Actualmente, por carecerse de indicadores sanitarios directos que midan aquellas, se emplean convencionalmente tasas que miden los riesgos de enfermar y de morir, en los distintos grupos poblacionales, valorándose en forma indirecta la salud, utilizando las tasas de mortalidad y secun. dariamente las de morbilidad, cuyos valores están afectos a una menor confiabilidad, debido a los frecuentes casos de subregistro de algunos rubros patológicos.

En el transcurso del siglo, se ha evidenciado que las condiciones de saisud del pais, expresadas en la forma antes expuesta, han experimentado cambios favorables, de acuerdo a una dinámica peculiar, en las que han alternado períodos a veces prolongados de tipo estacionario de las tasas, con lapsos en que éstas han registrado una tendencia notoriamente declinante, siendo esto último muy evidente entre los antos 1935 y 1955 y desde 1965 hasta la época actual.
El análisis de la tabla 2 es muy elocuente a dicho respecto, pudiendo presumirse que el proceso comprobado, guarda en general alguna,

Tabba 2.

Tasas de Mortalidad General e Infantil $1925-1981$

\begin{tabular}{|c|c|c|c|c|}
\hline & Mortalidad General & & Mortalidad Infantj] & $\% 0$ \\
\hline Año & $\% 0$ & Total & Neonztal & Tardía \\
\hline 1925 & 26.7 & 244.8 & 117.3 & 127.5 \\
\hline 1935 & 23.9 & 215.5 & 98.9 & 116.6 \\
\hline 1945 & 19.3 & 164.5 & 67.2 & 102.3 \\
\hline 1955 & 12.9 & 116.5 & 40.0 & 76.5 \\
\hline 1965 & 10.5 & 95.4 & 33.5 & 61.9 \\
\hline 1975 & 7.3 & 55.4 & 24.8 & 30.6 \\
\hline 1981 & 6.2 & 23.2 & 13.2 & 14.0 \\
\hline
\end{tabular}

Fuente: 1925-1965: Servicio Nacional de Salud e INE, 1970 (24) 1975-1981: Ministerio de Salud. 1982 (25).

aunque no siempre muy estricta relación con el progreso económico-social, cultural y sanitario del pais, habiendo sido ponderado $(26,27)$ el peso de cada grupo importante de factores eventualmente determinantes.

En lo que respecta especificamente al riesgo de 
morir en la edad infantil global, esto es en el grupo etario integrado por niños menores de 15 años de edad, la velocidad del descenso pertinente de las tasas, ha sido de distinta magnitud, como puede apreciarse en la tabla 3.

Tabla 3.

Variaciones absolutas $y$ porcentuales

de las Tasas de Mortalidad en menores de 15 a ños.

Chile. 1952-1975

\begin{tabular}{lrrc}
\hline $\begin{array}{l}\text { Tasa de } \\
\text { Mortalidad }\end{array}$ & $\begin{array}{c}1952 \\
\% 0\end{array}$ & $\begin{array}{c}1975 \\
\% 0\end{array}$ & $\begin{array}{c}\text { Velocidad de Reducción } \\
\%\end{array}$ \\
\hline Infantil & 117.8 & 55.4 & 52.9 \\
"Neonatal & 43.7 & 24.8 & 43.2 \\
"Tardía & 74.1 & 30.6 & 57.6 \\
$1-2$ años & 32.2 & 4.1 & 87.3 \\
$3-4$ años & 6.4 & 1.3 & 79.0 \\
$5-9$ años & 2.0 & 0.6 & 69.8 \\
$10-14$ años & 1.7 & 0.5 & 67.6 \\
\hline
\end{tabular}

Fuente: Kaempffer, A.M. (Ref, 28).

Los progresos reseñados, en lo referente al significativo descenso advertido en el último tiempo en las tasas antes consideradas, son concordantes en alguna medida con lo acaecido con las tendencias asimismo descendentes comprobadas entre 1955 y 1981 (28) en la mortalidad materna $(3.2 \%$ a $0.6 \%$ o) y perinatal $(52.7 \%$ a $18.8 \% 0)$.

En concomitancia con los cambios ocurridos en las tasas antes reseñadas, se ha registrado (29) asimismo una modificación sustancial en la estructura pertinente de aquellas, en particular en el primer afto de vida, siendo ello especialmente manifiesto en to acontecido con el descenso de la prevalencia especifitica de Enteritis y Enfermedades Diarreicas, y Respitatorias e Infecciosas, las que en conjunto en 1970 , constituyeron el $60.4 \%$ de los decesos en menores de un $a \tilde{n} \sigma_{2}$ en tanto que en 1979 alcanzaron sólo al $37.8 \%$. Por otra parte, las afecciones relacionadas con la morbilidad perinatal $y$ las congénitas, han registrado un incremento relativo desde $25.2 \%$ a $45.3 \%$ en idéntico período (30).

Sin duda, la gran disminución comprobada en la desnutrición, (aproximadamente un $8 \%$ en la última década) verdadero sustrato de una fracción importante de los decesos acaecidos en la menor edad, condiciona en singular medida algunos de los fenómenos antes mencionados.

La información correspondiente derivada de la Investigación Interamericana en la Niñez (31) ya reveló que la desnutrición, como causa básica o asociada, al inicio de la década de los años 70 , llegó a presentarse en Chile en cerca del $38.1 \%$ de las muertes ocurridas en menores de 5 años de edad, y dada la menor prevalencia actual, dicha cifra debe razonablemente haber descendido en forma significativa. Un hecho similar puede ocurrir respecto al bajo peso de nacimiento, que aún alcanza a cerca del $10 \%$ de los recién nacidos.

A la luz de las premisas expuestas, es comprensible que en el contexto internacional, los indicadores que ponderan el riesgo de morir en Chile, en el niño menor de un año, sean comparativamente satisfactorios, en especial en lo que respecta a países de la Región de las Américas, según se expone en la tabla 4.

Tabla 4.

Tasas de Mortalidad infantil, en países seleccionados en años tecientes. 1978-1981.

\begin{tabular}{lcc}
\hline País & Año & $\begin{array}{c}\text { Tasa } \begin{array}{c}\text { Mortalidad Infantil } \\
(\% o)\end{array} \\
\end{array}$ \\
\hline & 1979 & \\
Japón & 1978 & 8.0 \\
Suecia & 1979 & 9.6 \\
Francia & 1979 & 12.9 \\
Reino Unido & 1979 & 13.0 \\
Estados Unidos N.A. & 1978 & 14.7 \\
Alemania Federal & 1979 & 16.0 \\
Israei & 1978 & 16.2 \\
Jamaica & 1978 & 22.3 \\
Costa Rica & 1979 & 22.3 \\
Cuba & 1978 & 22.5 \\
Polonia & 1981 & 27.2 \\
CHILE & 1979 & 33.7 \\
Venezucla & 1978 & 40.8 \\
Argentina & 1978 & 44.1 \\
México & & \\
\hline
\end{tabular}

Fuente: U.N. (Ref. 32).

La situación de salud infantil ulterior en el país. durante los períodos de la niñez y de la adolescencia, es considerablemente más favorable $y$ como se seffaló anteriormente (tabla 3), los riesgos pertinentes de moric егал a mediados de la década del 70 , del orden de $0.6 \% 0$ y $0.5 \% 0$ respectivamente. Por otra parte, los rubros nosológicos que condicionan la morbi-mortalidad en las épocas en referencia, son diferentes, adquiriendo especial jerarquia accidentes, principalmente viales, las afecciones endocrinas, los tumores, algunas enfermedades infecciosas, tales como fiebre tifoidea, hepatitis y parasitosis.

Asimismo cabe destacar que en Chile, como en otros medios, en el niño mayor, en el adolescente $y$ en el joven ( 33 a 35 ), adquieren notoria importancia los trastornos psico-emocionales que dificultan la adaptación normal a la vida familiar, escolar y comunitaria y se condicionan de este modo hábitos y actitudes anómalas, del tipo del inicio del alcoholismo, tabaquismo, y en general 
del abuso de drogas. Todo ello puede predisponer a la aparición de comportamientos conductuales patológicos, tales como vagancia, violencia, prostitución e infracción social juvenil (33 a 35).

En lo referente al periodo etario que se comenta, una mención especial debe hacerse de los trastornos ligados al comienzo de la pubertad y de] ciclo reproductivo, en especial como consecuencia de los cambios de roles y de valores de la sociedad contemporánea y de la común inexistencia de una adecuada educación sexual y orientación para la vida familiar. Todo ello predispone, tanto a la aparición de enfermedades de trasmisión sexual, como a la procreación no deseada y al aborto inducido clandestino (36), cuya frecuencia aunque disminuída, constituye aún un problema sanitario importante (37).

Situaciones ecológicas particulares, tales como las determinadas por las condiciones propias de la pobreza critica, las subsecuentes a los procesos de migración temporal o permanente y la nueva estructura familiar, en que se está perdiendo el rol protector que en las condiciones expuestas, ejercia la "familia extendida", otorgan una severidad especial a la problemática mencionada.

\section{PLANES Y PROGRAMAS INTEGRADOS DE SALUD INFANTIL}

En Chile existe una tradición de langa data, iniciada a comienzos del siglo, orientada a la protección biológica y social del niño, en particular de áquel perteneciente a estratos en que las condiciones económico-sociales son precarias. Es así como ya en 1901, un organismo filantrópico privado, el Patronato Nacional de la Infancia, estableció las "Gotas de Leche", que fueron unida. des asistenciales destinadas a proporcionar atención médica ambulatoria, dación gratuita de leche e impartir ensefianza de Puericultura (38).

En 1911, se estableció el Servició Médico EscoJar, que tuvo por misión el realizar la supervisión periódica del estado de salud del niño matriculado en las escuelas primarias fiscales (38).

Entre los años 20 a 50, fueron creadas instituciones hospitalarias que estuvieron a cargo de la atención infantil especializada, dependientes de la Beneficencia Pública (Hospitales Manuel Arriarán, Luis Calvo Mackenna y Roberto del Rio) que han estado desarrollando una muy valiosa labor de asistencia médica primordialmente curativa, en estrecha coordinación con las Cátedras de Pediatria de las Facultades de Medicina de las Universidađes y que han sido factor de emulación y apoyo en el desarrollo de Servicios Pediátricos Hospitalarios de alta calidad, en la metrópoli $y$ en las grandes ciudades de las provincias.
En 1924, la creación de la Caja de Seguro Obligatorio, en el contexto de la visión creadora del Profesor Dr. Exequiel González Cortés (Ley No 4054) y a través de su Departamento de Servicio Médico, significó un- avance relevante en el otorgamiento de beneficios económicos y bioló. gicos a una fracción de la población obrera. Entre estos últimos, se incluía la protección de la gestante y del lactante hasta el octavo mes de vida, la garantía de reposo maternal y el aporte del auxilio de lactancia equivalente al $12.5 \%$ del subsidio de maternidad (38).

Ulteriormente el Servicio Nacional de Salubridad, originado en el Código Sanitario del año 1918, tuvo responsabilidad en la implementación de las disposiciones contempladas en el Título II "Del Bienestar del Niño y de la Madre", estipulados en el Código Sanitario promulgado en 1931 en base al D.F.L. No 226 (38). Con posterioridad, en 1938 y por iniciativa del Ministro de Salubridad, Previsión.y Asistencia Social de esa época, Dr. Eduardo Cruz Coke, fueron dictadas las leyes No 6154 ("Medicina Preventiva") y No 6233 ("Madre y Nirlo") que otorgaron beneficios de atención médica a la embarazada y a su hijo, quien recibió asimismo alimentación suplementaria hasta el $2^{\circ}$ año de vida (39).

Consecutivamente fue creado el Consejo de Defensa del Niño, institución đe protección social y tutela, y por disposiciones de la Ley No $7.200 \mathrm{y}$ D.F.L. 6/4.817, en 1942, la Dirección General de Protección de la Infancia y Adolescencia, que se responsabilizó del otorgamiento de servicios integrales de atención durante la gestación y la minoridad, abarcando asimismo la asistencia de la irregularidad biológica y social, en casos de indigencia económica. (39).

Contemporáneamente, en 1942 entran en funcionamiento las "Unidades Sanitarias" dependientes de la Dirección General de Sanidad y creadas con el apoyo del "Instituto Interamericano de Salubridad de los Estados Unidos". En los centros mencionados se prestó atención preferentemente preventiva a grupos familiares no asistidos por otros sistemas previsionales que a la sazón ya extendian sus beneficios a un gran sector de trabajadores públicos, privados e independientes. Un interesante estudjo realizado en aquella época (40) destacó los progresos en la atención infantil institucionalizada la que alcanzaba a cubrir hasta $1 / 3$ de la población total, que recibió servicios médico-sanitarios que contribuyeron a lograr un descenso importante de las tasas de mortalidad infantil entre sus beneficiarios.

La creación, en 1952, del Servicio Nacional de Salud (SNS) con la promulgación de la Ley No10.383 (39), estando a cargo del Ministerio del ramo, el Dr. Jorge Mardones Restat, permitió 
ampliar apreciablemente la cobertura asistencial integral de madres y de niños, en el contexto de la atención de la salud de la familia y llegándose a cubrir hasta $2 / 3$ de la población del país. Para ello se aunaron y catalizaron los recursos de diversa índole que en alta proporción procedieron de las instituciones antecesoras al Servicio y a las que ya se ha hecho referencia.

El SNS ha constituido el hito de mayor trascendencia en la historia sanitaria de Chile y su experiencia se ha proyectado ampliamente al extranjero. Entre las características más relevantes đe la institución, deben destacarse la programación de sus actividades en el marco de referencia de planes de salud realizados en articulación intersectorial, aunque no siempre en la plenitud deseada. Constituyen en el SNS beneficios específicos del grupo maternoinfantil y de la familia, la extensión aún en áreas marginales, de actividades susceptibles de realizarse en forma simultánea. Entre éstas se destaca la atención médica y dental integral, la vigilancia continua (39) de la normalidad durante el ciclo reproductivo. (Atención técrica del parto $-92 \%$ en $1981 \cdots$ y cuidado de la salud perinatal), la supervisión durante el período del crecimiento y desarrollo, la atención especial de los casos de mayor riesgo $(37,41)$, los programas amplisimos de vacunaciones, la educación higiénica, la orientación para la vida familiar, incluyendo la información y servicios si asi procediera, de regulación de la fecundidad, para promover la paterniđad responsable. Estas últimas actividades que se estaban desarrollando en el país inicialmerte bajo la orientación de la entidad privada "Asociación de Protección de la Familia", filial de la Asociación Internacional de Planificación Familiar (lPPF), fueron institucionalizadas oficialmente en el SNS a partir de 1965 (36), con el objetivo de contribuir fundamentalmente a disminuir los riesgos que presenta el aborto inducido, la maternidad temprana y no adecuadamente espaciada y la elevada paridad, tópicos sobre los que existe una valiosa experiencia nacional (27 a 29 , 37) e internacional $(13,31,41,42)$ acerca de los beneficios que cabe esperar con las acciones referidas. Todo ello en el entendido que se asigne pleno respeto por la libre y digna decisión parental en conformidad con las políticas nacionales que han estado vigentes (43).

Una mención especial cabe hacer de las actividades que se cumplen en el Programa Nacional de Alimentación Complementaria (PNAC) que ha permitido el suministro, en 1980, de 25,195 toneladas de leche y de alimentos proteicos a embarazadas, nodrizas y párvulos. Dicha cobertura, como se señala en la tabla 5 , ilustra acerca del incremento progresivo de la extensión del programa mencionado, el que es la expresión de una política previa de larga data.

Tabla 5.

Distribución de Leche y Sustitutos Lácteos $195 \$-1980$

\begin{tabular}{cc}
\hline AÑO & VOLUMEN (TONS.) \\
\hline 1955 & 1.400 \\
1960 & 8.400 \\
1965 & 9.166 \\
1967 & 18.146 \\
1975 & 18.452 \\
1980 & 25.351 \\
\hline
\end{tabular}

Fuente: Ref. (29), con adaptación.

Al programa mencionado, debe añadirse el apoyo técnico. a las actividades de protección alimentaria que realiza la Junta Nacional de Auxitio Escolar y Becas (300.000 almuerzos y 750.000 desayunos escolares $\}$, y de diversas instjtuciones de cuidado infantil comunitario o perte. necientes a otras instituciones (44 a 46). Las actividades antes reseñadas se complementan con los programas de Fomento de la lactancia Materna de contenido básicamente educativo (47) y de Recuperación de Desnutridos (45), a cargo de una Corporación privada ( CONIN"), que se desarrolla en 32 centros de atención infantil y familiar, ubicados a lo largo de todo el país, y donde se cumple una labor muy encomiable.

Por otra parte, entre las actividades de relieve realizadas por el Sector Salud, y que benefician al niño y a la familia, debe hacerse referencia a las cada vez más extensas acciones de seguridad social, traducidas en la ampliación de las asignaciones familiares y que incluyen ya desde 1956, la protección de la gestante y que progresivamente se han extendido para cubrir a todos los niños menores de 8 años cuyas familias se encuentren en condiciones de indigencia.

Finalmente, deben destacarse los progtamas que bajo la tuición técnica de diversas instituciones de gobierno y con importante participación comunal, llevan a cabo una miríada de entidades de protección al niño, a la madre y a la familia, y entre las que cabe referirse a "CEMA. CHILE", a través de los Centros de Madres y que dirige los Hogares para adolescentes, para embarazadas y para madres campesinas, los Centros de cuidado infantil diurnos de la Corporación de Ayuda al Menor "CORDAM" y de niños lisiados de la Corporación de Ayuda al Niño Limitado "COANIL"., la Fundación de Jardines Infantiles y el "Servicio Nacional del Menor", creado en 1979 por D.L. No 2465 y que desarrolla en el ámbito del Ministerio de 
Justicia y en adecuada coorđinación intersectorial, relevantes actividades. preventivas, de diagnóstico, tratamiento y rehabilitación, en torno a niños y familias en riesgo biológico y/o social ("irregulares") y las que en su conjunto con las mencionadas anteriormente, configuran el vigente Plan Nacional de Menores (48 y 49).

En fecha reciente, se han promulgado disposiciones legales de acuerdo a las cuales se introducen modificaciones en las instituciones antes descritas de Salud (D.L. 2763) y de Previsión (D.L. 3626) y las que han significado innovaciones estructurales, administrativas y funcionales (50) que se están llevando a cabo en forma progresiva. En sintesis, de lo que se ha tratado, es de promover el fortalecimiento del Ministerio de Salud, desde los puntos de vista normativo, de control y de evaluación, descentralizando la ejecución de las acciones mismas a través de 27 Servicios de Salud, los que en el contexto de las Secretarías Regio. nales Ministeriales, aplican la política del "Sistema Nacional de Salud", de acuerdo a la realidad local. Asimismo se ha favorecido la apertura de un "extrasistema" previsional y de salud, en base al financiamiento privado de las prestaciones. Cabe destacar que las modificaciones legales establecidas recientemente, no deben alterar en su esencia el propósito básico del sistema, que reside en la extensión y mayor eficiencia del nivel primario de atención, que en la actualidad recae mayormente en el ámbito del quehacer operacional comunal.

\section{ROL DE LA COLABORACION INTERNACIONAL}

La colaboración internacional en relación a la salud en general y en particular de la del niño y la familia, ha tenido en Chile siempre una significativa importancia. Esta ha sido brindada por distintas instituciones, sean de tipo privado, gubernamental u organizaciones de cooperación multilateral, pertenecientes mayormente al sistema de Naciones Unidas (51).

Las actividades colaborativas en referencia, en el contexto de la salud infantil, han significado en ciertas ocasiones un apoyo directo a las acciones específicas nacionales pertinentes, en tanto que en otras, el beneficio para la salud del niño ha sido obtenido como resultado de programas cooperativos de índole sanitaria global, tal el caso de los realizados fundamentalmente en torno a la higiene de la vivienda, el saneamiento ambiental y la erradicación de enfermedades epidémicas que pueden afectar a toda la población. Por obvias razones, se analizará en el presente trabajo, primordialmente la primera de las situaciones antes expuestas, estableciendo las connotaciones corres- pondientes tanto a la agencia colaboradora, como a la época, el área y la metodología de su accionar.

Entre las instituciones de colaboración privada de mayor relieve en el campo de la salud infantil, se ha contado en Chile con el apoyo de las Fundaciones Rockefeller, Ford y Kellog, las que han desarrollado sus actividades cooperativas fundamentalmente en el ámbito de la docencia e investigación básica y operacional.

A dicho respecto cabe destacar la importante contribución de la Fundación Rockefeller en la creación en 1944, de la Escuela de Salubridad, cuyo Departamento de Salud Maternoinfantil ha tenido una gran trascendencia en 1a formación académica de profesionales nacionales y asimismo latinoamericanos que se han desempeñado con efjeciencia en los programas y servicios de protección infantil y familiar, $y$ en las actividades correspondientes de docencia universitaria (51).

En el ámbito de la nutrición y la reproducción humana, ha sido relevante el apoyo de la Fundación Ford, así como la Fundación Kellog, en el campo de la capacitación en atención pediátrica, tanto especializada, a través del programa de residencias, como con la relacionada con la atención primaria.

Por lo que respecta a la colaboración de índole gubernamental, fundamentalmente su finalidad ha residido en el pasado en el fortalecimiento de servicios asistenciales, tal el caso del decisivo. aporte del Servicio Cooperativo Interamericano de los Estados Unidos de América en la creación de las "Unidades Sanitarias", en las que tuvo especial relieve el desarrollo de actividades de salud maternoinfantil que favorecieron a grupos farniliares que no estaban afiliados a organismos de previsión (51).

Cabe asimismo en este acápite hacer referencia de la amplia cooperación que el pais ha recibido de la "Agencia Internacional para el Desarrollo" del gobierno de los Estados Unidos de América, (AID), en tomo básicamente a los programas alimentario-nutricionales y de salud familiar que se han llevado a cabo en el país (51).

Finalmente debe hacerse mención en el rubro en análisis, del apoyo docente prestado por el Centro Intemacional de la Infancia, de París, en el área de la pediatría social, otorgando becas para el perfeccionamiento científico de profesionales chilenos en el extranjero. Asimismo el Centro mencionado ha proporcionado oportunidades a técnicos nacionales para participar en la Investigación cooperativa de alto nivel sobre "Estudios Coordinados de Crecimiento y Desarrollo Infantil".

Las Agencias especializadas de Naciones Unidas y de un modo especial las Organizaciones Mundial (OMS) y Panamericana de la Salud (OPS) son sin 
duda las que han brindado a Chile una cooperación de mayor magnitud y continuidad.

A dicho respecto, cabe destacar el rol de insustituible significado que desde comienzos del siglo ha prestado a Chile y a América la Oficina Sanitaria Panamericana $(51,52)$, Secretariado Regional de la OMS y OPS. Dicha institución ha ajustado racionaimente sus actividades cooperativas de acuerdo a una dinámica que ha contem. plado las necesidades y el requerimiento de los paises, en concordancia con la problemática sanitaria global de las Américas, favoreciendo de este modo el intercambio de experiencias y la cooperación técnica entre los países mismos. Por otra parte, debe hacerse mención a que la labor de la Ofícina Sanitaria Panamericana en el transcurso de las últimas décadas, ha pasado de la asistencia en situaciones coyunturales en el corto y mediano plazo, tales como erradicación de enfermedades cuarentenables e infecciosas, saneamiento rural (con el apoyo del Banco lnteramericano de De. sarrollo), nutrición y especialmente formación de recursos humanos, a la cooperación técnica conjunta a largo plazo, sirviendo de apoyo a los servicios de salud, con énfasis en el desarrollo de las actividades de salud infantil y familiar $y$ en el contexto sucesivamente de los Planes de Salud Continentales (1961-1971 y 1972-1981) y de su actualización en la IV Reunión Especial de Ministros de Salud de las Américas (53 a 55).

En el contexto expuesto, debe hacerse mención de la importante contribución de la Oficina Sanjtaria Panamericana al desarrollo de Cursos avanzados, de proyección latinoamericana, entre otros el de Pediatría Clínica y Social y el de Atención Primaria de Salud Infantil, en conjunto con Departamentos de Pediatría universitarios, y el de Administración en Salud Maternoinfantil a nivel de la Escuela de Salubridad.

En la década de los años 70, la Organización Panamericana de la Salud, en conjunto con UNICEF y el Centro Latinoamericano de Demografia (CELADE) y contando con la implementación financiera del Fondo de Naciones Unidas para Actividades de Población (FNUAP), prestó asesoría continua durante la ejecución de los Proyectos a gran escala de "Extensión de Servicios de Salud Maternoinfantil y Bienestar Familiar" (PESMIB) $y$ de Atención Matemoinfantil Rural (PRUMIN) y que se tradujeron en el fortalecimiento de las actividades en desarrollo en el país, de salud maternoinfantil $y$ de regulación de la fecundidad, en el contexto de las políticas gubernamentales vigentes (43). La realización exitosa de los proyectos mencionados (56 a 57), fue una circunstancia propicia para el desarrollo de programas similares en la mayoría de los países de
Latinoamérica, $y$ en cuya génesis tuviéramos el privilegio de participar (58).

Finalmente, cabe referirse a la valiosa cooperación que OPS/OMS prestó a Chile en coordinación con centros universitarios del pais, en torno a la Investigación de la Mortalidad en la Niñez (31), proyecto cooperativo de alcance continental y cuyos hallazgos han contribuido al perfeccionamiento de Ios programas nacionales de salud in. fantil. También en el área de investitación debe mencionarse la oportunidad que OMS ha prestado al país, para participar en el Estudio Cooperativo sobre "Modelos de Amamantamiento" y que han contribuido a fortalecer el programa pertinente de promoción de la lactancia materna, que orienta el Ministerio de Salud (59).

El Fondo de las Naciones Unidas para la Infancia (UNICEF), ha sido otra de las Agencias de Naciones Unidas cuya colaboración constante $y$ eficaz ha contribuido a la promoción de la salud y el bienestar del niño y de la familia, tanto por su participación en los proyectos cooperativos interagenciales antes mencionados, como en los que han significado-apoyo de actividades especificas en el campo de la alimentación infantil $y$ el fomento de la lactancia materna, de la implementación de unidades de atención pediátrica en diversos niveles asistenciales, desde los de alta especialización (Centros de Prematuros y de Atención Perinatal) hasta los de las postas rurales y de toda la gama de entidades asistenciales de protección del niño, y a los que anteriormente se hiciera referencia (Jardines Infantiles, Núcleos Escolares, Centros de Tutela de Minoridad) y que en su conjunto configuran los programas del Plan Nacional de Menores de Chile (48). Se otorga en estos casos un énfasis especial al fortalecimiento de las acciones de estimulación temprana psico--sensorial (60), en especial a nivel de las áreas de deprivación socio-cultural, propia de los medios de pobreza crítica en que actúan los proyectos y para los cuales se ha recibido asimismo el apoyo del Instituto Interamericano del Nif̄o (IIN), Agencia Especializada de la Organización de Estados Americanos (OEA) (61), y cuya finalidad reside en la Protección Integral de la Matemidad, el Niño y la Familia:

En años recientes y en concordancia con las Resoluciones de la III y IV Reuniones Especiales de Ministros de Salud $(53,54)$, Chile, como los restantes países de Latinoamérica y el Caribe, han orientado sus planes nacionales sanitarios hacia el Jogro de una cobertura universal programática, alcanzando las áreas de mayor marginalidad y ruralidad, y utilizando para ello y en un contexto intersectorial y con decidida participación comunitaria, la metódica de la atención primaria (62), la 
que lleva implícita un componente destacado de protección de la salud infantil y familiar. A este respecto debe destacarse que los favorables resultados obtenidos por el pais (30), al término del 3er. Plan Decenal de Salud (54), en particular en el ámbito de la salud del niño, permiten tener un razonable optimismo acerca del cumplimiento de los objetivos que el Plan de Acción (23) pertinente contempla, y que apuntan a la obtención de Salud para Todos en el Año 2000. Para este propósito y en forma continua, se ha estado contando con la cooperación internacional de las Agencias antes mencionadas, considerando que su apoyo en áreas críticas será insustituible para llevar a feliz término $\tan$ trascendente tarea.

Asimismo, será decisivo, como siempre lo fue en el pasado en oportunidades similares, el contar con la abnegación, entusiasmo y el talento inmerso en las Asociaciones Gremiales (Colegio Médico de Chile A.G.) y Cientificas (Sociedad Chilena de Pediatria), en concordancia con su permanente vocación de servicio y de humanismo (63).

\section{RESUMEN}

Las condiciones de salud en Chile, han experimentado en las últimas décadas progresos significa- tivos, los que se han traducido fundamentalmente en tasas de tendencia declinante de mortalidad infantil y parvularia, las que alcanzaron en 1981 a $27.2 \%$ 。 y $1.16 \%$ 。 respectivamente.

La situación expuesta es el resultado de una miríada de factores, la mayoría de ellos ligados al grado de desarrollo global alcanzado por el pais. En este contexto, se considera que la creación y el funcionamiento razonablemente satisfactorio de una estructura sanitaria nacional, ha tenido una singular trascendencia, la que ha permitida la rea. lización y con amplia cobertura, de actividades integrales de salud, con un relevante componente de asistencia infantil $y$ contando con una valiosa participación de las familias y de la comunidad.

Se estima que la colaboración internacional bi y/o multilateral brindada en el país, en particular la pertinente de las Agencias Especializadas de Naciones Unidas y en especial de las Organizacio. nes Mundial y Panamericana de la Salud y et UNICEF, ha sido valiosa por su apoyo catalítico a los Planes Nacionales de Salud que se han desarrollado en Chile y que apuntan en la actualidad a la obtención de Salud para Todos en el Año 2000, meta cuyo cumplimiento incrementará el bienestar infantil.

\section{REFERENCIAS}

1 Rosselot, J.: Salud Matemoinfantil en Latinoamérica. Bol. Of. Sarit. Panam. 70,5: 407-421, Washington, D.C. 1971

2Rosselot, J.: Child Health in Latin America and the Caribbean, in Borsh, S.J. and J. Atias (eds). Evaluation of Child Health Services. The Interface between Research and Medical Practice. DEW Publicanion. (NIH) 78-1066. Goverment Printing Office. Washington, D.C. 1975.

${ }^{3}$ Rosselor, J.: Situación de la Salud Infantil en Latinoamérica $y$ el Caribe $y$ el Rol del Instituto Interamericano del Niño (IIN). Informes Técnicos. IIN. Sección Salud No 7 . Mon tevideo, 1981.

4 Organización Mundial de la Salud.: Desarrollo Humano y Salud Pública. Serie de Informes Técnicos. No 485. Ginebra. 1972.

\$ Organización Mundial de la Salud.: Salud de la Madre y el Niño. Informe del Director General (A 32/1-3 de Abril de 1979). 32 Asamblea Mundial de la Salud. Ginebra. 7-25 de Mayo de 1979.

- Organización Mundial de la Saluat: Nuevas tendencias y métodos de asistencia matemoinfantil en Ios Servicios de Salud. Sexto In forme del Comité de Expertos de la OMS en Salud de la Madre y el Niño. Serie de Informes Técnicos No 600. Ginebra. 1976

${ }^{\top}$ Naciones Unidas.: Declaración Universal de los Derechos del Hombre. Lake Success. New York. 1979.

Instituto Interomericano del Niño.: Los derechos del Niño. Montevideo. 1961.

9 Naciones Unidas: Año Intemacional đel Niño. 106a Asamblea Plenaria. Resol. 31/169. New York. 1976.

10 Organización Mundial de la Salud.: 36а Asamblea Mundial de la Salud. Resol. WHA. 36/43. Ginebra. $197 ?$.
11 Nociones Unidas.: Asamblea General. Resol. 34/58. New York. 1978.

12 Fondo de las Naciones Unidas para la Infoncio. Situación de la Infancia en América Latina y el Caribe. Coordinación de Juan Carlos Terra. UNICEF. Oficina Regional para las Américas. Santiago de Chile. 1979.

13 Organización Panamericana de la Salud.: Condiciones de Salud del Njño en las Américas. Publicación Científica No 381 . Washington, D.C. 1979.

14 Banco Interamericano de Desamollo.: Progreso Económico y Social en América Latina Informe 1980-81: Washington, D.C. 1981.

1 S Ministerio de Salud.: Estrategias de Salud de Chile hasta el Año 2000. Santiago. 1981.

16 Ministerio de Satud.: Indicadores Biodemográficos. Dpto. de Planificación. Santiago. 1981.

17 Altimir. $0 .:$ La dimensión de la pobreza en América Latina. CEPAL, 1978.

1 Sanfuentes, A, y $H$. Lavados. Dimensiones de la pobreza en America Latina y el Caribe. LNICEF. Santiago de Chile. 1982.

19 Ministerio de Salud.: Chite. Situación de Salud. Santiago. 1978.

20 Grant, J.P.: Nuevo procedimiento para medir los progresos dél nivel de vida. Foro Mundial de la Salud (OMS) Vol. 2 No 3: 433-446. Ginebra. 1981.

21 Grant, J.P.' Estado Mundial de la Salud. UNICEF. New York. 1980.

22 World Health Assembly.: Resol. WHA 34. 36. Global Strategy for all by the year 2000 . Document A. 34.5. Geneva. 1980.

23 Organización Ponamericane de la Salud.: Salud para Todos en el Año 2000. Plan de Acción para la implementación de Estrategias Regionales. Documento 
Oficial No 179. Washington, D.C. 1982.

24 Senicio Nacionat de Salud e INE.: La Población de Chile. Santiago, 1970.

25 Ministerio de Salud.: Indicadores Bjodemográticos. Chile. 1972-1981. Santiago, 1982.

$26 \mathrm{Behm}$, H. Mortalidad Infantil y Nivel de Vida. Ediciones de la Universidad de Chile. Santiago, 1962.

27 Taucher, E.: La mortalidad infantil en Chilé. Notax de Población, Año VII, No 20: 35-71. CELADE. Santiago, 1979.

28 Kaempffer, A.M.: Fvolución de la Salud Maternoinfantil en Chile, 1952-1977. Rev. Méd. Chile. 105: 680. 1977 .

29 Raczynski, D. y C. Oyarzo.: ¿Por qué cae la tasa de Mortalidad Infantil en Chile? Coleccion de Estudios CIFPLAN 6 No 55. Santiago, 1981.

30 Ministerio de Salud.: Participación del Sector Salud en la Atención Integral del Menor. Santiago, 1978.

31 Puffer, R.R. y C.V. Serrano.: Características de la Mortalidad en la Niñez. Organización Panamericana de la Salud. Publicación Científica No 262. Washington, D.C. 1976.

32 United Nations: Pepulation and Vital Statistics Report. Data available as of January 1981. Statistical Papers. Series A. Vol. XXX No 1. New York.

33 Rosselot, J.: La Salud del Adolesccnte $y$ del Joven en América Latina $y$ el Caribe. Boletín de la Oficína Sanjtaria Panamericana. Vol. 83, 84: 295-309. Washington, D.C. 1977.

34 Organización Mundial de la Salud.: Necesidades de Salud de los Adolescentes. Serie de lnformes Técnicos. No 568 . Ginebra. 1977.

35 Kampffer, A.M.: Mortalidad y Morbilidad del Adoles cente en Chile. Pediatría. Vol. 20 No 1: 17-30. 1977.

${ }^{36}$ Rosselot, J. y cols,: Informe sobre Politica del Servicio Nacional de Salud para regular la natalidad en Chile. Rev. Méd. Chile. 94: 774-750. 1966.

${ }^{37}$ Molina, $R$.: Elementos de Salud Materna y Perinatal. Ed. Andrés Bello. Santiago. 1981.

38 Rosselot, J.: Problemática de la Protección Matemainfantil en el Servicio Nacional de Salud de Chile. Pediatría. Vol. 8, No 3: 222-231, 1965

39 Vio Valdivieso, $F_{,}$: El Derecho a la Salud en la Legislación Chilena. Editorial Jurídica de Chile. Santiago. 1956.

40 Mardones, $F$. A. Horwitz, 'G. Adriasolo, $O$. A vendaño $y J$. Meneghello.: Atención Matemoinfantil en Chile. Servicio Nacional de Salud. San tiago. 1954.

41 World Health Organization.: Risk Approach for Maternal and Child Health Care. WHO. Offset Publication. No 39. Geneva. 1978.

42 Puffer, R.R. y C,V, Serrano,: El peso al nacer, la edad inatema y el orden de nacimiento. Tres importantes factores determinantes de la Mortalidad Infantil. Organización Panamerjcana de la Salud. Publicación Cienlifica No 294. Washington, D.C. 1975.

43 Odeplan.: Presidencia de la República de Chile. Política Poblacional en el Plan Nacional de Desarrollo (1978-1983). Santiago. 1978.

$\$ 4$ Monckeberg, F.: Cambios en la Nutrición Infantil en Chile. VT Reunión del Consejo Directivo de la Asociación Latinoamericana de Academias Nacionalos de Medicina y Conferencias Médicas Latinoamericanas.
Instituto de Chile. Academia de Medicina. Santiago. 1980.

45 Monckeberg, $F_{.:}$Crear para compartir. Compartir para seguir creando Editorial Andrés Bello. Santiago. 1980.

46 González, N. y H. Sánchez.: CONPAN y el Problema Nutricional de Chilc. Colegio Médico de Chile. Cuademos Médico-Sociales. Vol. XXI, NO4: 7 a 23. Santiago. 1980.

47 Ministerio de Salud: Manual de Fomento para la Lactancia Mate ma. Santiago. 1979.

48 Ministerio de Justiciat.: Plan Naçional para Menores. Santiago. 1978.

${ }^{49}$ Ministerio de Justicia, Políticas y Acciones dirigidas al Menot en Chile. Santiago. 1981.

50 Sanchez, H.: Análisis Evolutivo del Sistema de Salud Chileno. Colegio Médico de Chile. Cuademos Médico-Sociales. Vol. 22, No 1: 10 a 19. Santiago. 1981.

51 Juricic, B.: Chile y las Organizaciones Intemacionales de Salud. Rev. Méd. de Chile. Vol. 105, No 10; 674-679. 1977 .

52 Rosselor, $f^{*}$ La Organización Panamericana de la Salud y los Programas de Salud Matemoinfantil y Bienestar Familiar en Latinoamérica y el Caribe. Organización Panamericana de la Salud. Documento Mimeografiado. Washington, D.C. 1975.

53 Organizaclón Panamericana de la Salud.: Reurión de Ministros de Salud. Grupos de Estudio. Informe Final. Documento TFH/14 (Esp.), 20 de Abril, 1963. Washington, D.C. 1963.

54 Organización Panamericana de la Salud.: Plan Decenal de Salud para las Anéricas. Documento Oficial No 118 Washington, D.C. 1973.

55 Organización Panamericana de la Salud: IV Reunión Especial de Ministros de Salud de las Américas. In. forme Final y Documentos de Referencia. Documento Oficial No 155. Washington, D.C. 1978.

56 Ministerio de Salud.: Informe de Evaluación Final del Proyecto de Extensión de Servicios de Salud Maternoinfantil y de Bienestar Familiar (PESMIB). Santiago. 1978.

57 Ministerio de Saluet: Informe de Evaluación Final đel Proyecto de Atención Matemoinfantil Rural. Santiago. 1981.

58 Rosselot, J.: Misión cumplida en la Oficina Sanitaria Panamericana, (1968-1978). Organización Panamericana de la Salud. Documento Mimeografiado. Washington, D.C. 1978.

59 World Health Orgonizasion.: Contemporary Patterns of Breast-Feeding. Report on the WHO Collaborative Study on Breast-feeding. Geneva. 1981.

60 Montenegro, $\boldsymbol{H}$. $y$ cols.: Estimulación Temprana. Importancia del Ambiente para el Desarrolla del Niño. UNICEF. Santiago. 1981.

61 Sajón, R.: Boletín del Instituto Interamericano del Niño (50 Aniversario del IIN), No 200:3-57. Montevideo. 1977.

62 Alma Ata. 1978.: Atención Primaria de Salud. Informe de la Conferencia Internacional sobre Atención Primaria de la Salud. Alma Ata (URSS), 6.-12 de Septiembre de 1978. Orgarización Mundial de la Salud. Ginebra. 1978.

${ }^{63}$ Ariztía, A.: Breve reseña histórica de la Pediatría en Chule. Rev. Chilena de Pediatría. 50: 97-104, 1979. 